\title{
Bulk universality of sparse random matrices
}

\section{Citation}

Huang, Jiaoyang, Benjamin Landon, and Horng-Tzer Yau. 2015. “Bulk Universality of Sparse Random Matrices." Journal of Mathematical Physics 56 (12) (December): 123301. doi:10.1063/1.4936139. .

\section{Published Version}

doi:10.1063/1.4936139

\section{Permanent link}

http://nrs.harvard.edu/urn-3:HUL.InstRepos:32189254

\section{Terms of Use}

This article was downloaded from Harvard University's DASH repository, and is made available under the terms and conditions applicable to Other Posted Material, as set forth at http:// nrs.harvard.edu/urn-3:HUL.InstRepos:dash.current.terms-of-use\#LAA

\section{Share Your Story}

The Harvard community has made this article openly available.

Please share how this access benefits you. Submit a story.

Accessibility 


\title{
Bulk universality of sparse random matrices
}

\author{
Jiaoyang Huang, ${ }^{\text {a) }}$ Benjamin Landon, ${ }^{\text {a) }}$ and Horng-Tzer Yau ${ }^{\text {a) }}$ \\ Department of Mathematics, Harvard University, Cambridge, Massachusetts 02138, USA
}

(Received 12 July 2015; accepted 6 November 2015; published online 1 December 2015)

\begin{abstract}
We consider the adjacency matrix of the ensemble of Erdôs-Rényi random graphs which consists of graphs on $N$ vertices in which each edge occurs independently with probability $p$. We prove that in the regime $p N \gg 1$, these matrices exhibit bulk universality in the sense that both the averaged $n$-point correlation functions and distribution of a single eigenvalue gap coincide with those of the GOE. Our methods extend to a class of random matrices which includes sparse ensembles whose entries have different variances. (C) 2015 AIP Publishing LLC. [http://dx.doi.org/10.1063/1.4936139]
\end{abstract}

\section{INTRODUCTION}

The universality of the spectral statistics of random matrices has been a central subject since the pioneering works of Wigner (Ref. 34), Gaudin (Ref. 23), Mehta (Ref. 27), and Dyson (Ref. 7). The first such universality result is the global semicircle law of Wigner which states that under some weak moment conditions, the empirical eigenvalue distribution of a matrix with i.i.d. entries converges weakly to the deterministic semicircle law,

$$
\lim _{N \rightarrow \infty} \frac{1}{N} \sum_{i=1}^{N} \delta_{\lambda_{i}} \rightarrow \rho_{\mathrm{sc}}(E):=\frac{1}{2 \pi} 1_{\{|E| \leq 2\}} \sqrt{4-E^{2}},
$$

in the appropriate scaling.

The Wigner-Dyson-Gaudin-Mehta conjecture, or "bulk universality" conjecture, states that the local statistics of the eigenvalues of random matrix ensembles should be universal in the sense that they depend only on the symmetry class of the random matrix ensemble but are otherwise independent of the law of the matrix entries. Here, local statistics refer to the behaviour of the eigenvalues in the scaling when their typical distance is of order 1.

A prominent class of random matrices are Wigner matrices. These matrices have independent centered entries with a uniform subexponential decay condition and identical variances. The Wigner-Dyson-Gaudin-Mehta conjecture for Wigner matrices was recently established in a series of papers (Refs. 11, 8, 13, 20,10, and 17) for all symmetry classes. Parallel results in various cases were obtained in Refs. 33 and 32.

The conclusion of Refs. 11, 8, 13, 20, 10, and 17 was that Wigner matrices, and even the wider class of generalized Wigner matrices in which the variances of the entries may differ, exhibit bulk universality in the following two forms. The first is that the $n$-point correlation functions are universal after averaging over a small energy window. The second is that the distribution of the eigenvalue gaps with a fixed label is universal. For Wigner matrices, the universality of the averaged $n$-point correlation functions is equivalent to the universality of a local average of eigenvalue gaps. However, there is no rigorous mathematical relation between the universality of the eigenvalue gaps with a fixed label and the universality of the $n$-point correlation functions at a fixed energy. Universality at a fixed energy has recently been established for all symmetry classes in Ref. 5, but we will not be concerned with this type of convergence in this work.

Wigner matrices were originally introduced in Ref. 34 by Wigner to model the spectra of heavy atoms and are widely used to model systems in which all elements strongly interact with one

\footnotetext{
a)Electronic addresses: jiaoyang@math.harvard.edu; landon@math.harvard.edu; and htyau@ math.harvard.edu
} 
another. However, for systems in which the links between different elements are broken, a better description is offered by the so-called sparse (or dilute) random matrices which have an average of $p N$ nonzero elements per row, for $p \ll 1$.

Aside from theoretical physics models, sparse random matrices also arise in graph theory in the study of sparse random graphs. Perhaps the simplest example is the Erdôs-Rényi ensemble which consists of a random graph on $N$ vertices in which each edge is chosen independently with probability $p$. The adjacency matrix of this graph is called the Erdős-Rényi matrix. The Erdős-Rényi matrix has typically $p N$ nonzero entries in each column and is sparse if $p \ll 1$. As the matrix entries take values 0 or 1 , the mean of the entries is not 0 . Ignoring the nonzero mean for the moment, the Erdős-Rényi matrix can be viewed as a singular Wigner matrix, as the probability distribution of the matrix elements is highly concentrated around 0 . The singular nature of this ensemble can be expressed by the fact that the $k$ th moment of a matrix entry is bounded by

$$
N^{-1}(p N)^{-(k-2) / 2} \text {. }
$$

When $p \ll 1$, this decay in $k$ is much slower than in the case of Wigner matrices.

It was conjectured in Ref. 21 that for sparse random matrices, there exists a critical value $p_{c}>1$, such that for $p N>p_{c}$, the bulk eigenvalues are strongly correlated and are characterized by GOE/GUE random matrix statistics; for $p N<p_{c}$, the eigenvalues remain uncorrelated and follow Poisson statistics. This conjecture is supported by a wealth of numerical simulations (Refs. 21 and 25) and a nonrigorous supersymmetric approach (Refs. 22 and 28). The best rigorous result in this direction was obtained in Refs. 9 and 8 and asserts that if

$$
p N \geq N^{2 / 3+\epsilon},
$$

then the averaged $n$-point correlation functions of the Erdôs-Rényi ensemble coincide with the GOE.

In the present work, we prove that in the regime

$$
p N \geq N^{\epsilon},
$$

the local statistics of the Erdôs-Rényi ensemble exhibit bulk universality. In addition to proving that the averaged $n$-point correlation functions coincide with the GOE, we also prove the universality of the eigenvalue gaps with a fixed label. To further place the present work in context we recall the three-step strategy developed in Refs. 11, 8, 13, 20, 10, and 17 for proving universality for Wigner matrices.

(1) Establish a local semicircle law controlling the number of eigenvalues in windows of size $\log (N)^{C} / N$.

(2) Analyze the local ergodicity of Dyson Brownian motion (DBM) to obtain universality for Wigner ensembles with a small Gaussian component.

(3) A density argument comparing a general Wigner matrix to one with a small Gaussian component.

For an overview of this three-step strategy and more details, we refer the reader to Ref. 16 . The local semicircle law for sparse random matrices in regime (1.4) was established in Ref. 9. However, in Ref. 8, steps (2) and (3) were only completed for sparse random matrices in regime (1.3).

The key input from step (1) into step (2) is a high-probability a priori bound on the eigenvalue locations which is a corollary of the strong semicircle law. In the case of Wigner matrices, this bound is optimal and it allows one to conclude that local equilibrium is reached in times $t=N^{\epsilon} / N$. Sparse random matrices do not obey as strong as a semicircle law and so the time to equilibrium found in the work in Ref. 8 was much longer. Moreover, due to the slow decay of the third moment, the approximation in step (3) is not as strong in the case of Wigner matrices and so could not be used for the large times required by step (2). These two factors led to condition (1.3) of Ref. 8 .

In the recent work in Ref. 26, the optimal time of Dyson Brownian motion to local equilibrium was established for a wide class of initial data (see Ref. 15 for related results on DBM with general initial data). Using this as an input we will prove that DBM reaches local equilibrium in the optimal time $t=N^{\epsilon} / N$ when the initial data are sparse random matrix. For the comparison of correlation functions, step (3) was obtained in Ref. 8 via a Green function comparison theorem. In this paper, we will use a lemma of Ref. 6 which asserts continuity of DBM when viewed as a matrix 
Ornstein-Uhlenbeck process. It is interesting to note that this continuity lemma provides a very convenient tool for step (3) in the sparse setting whenever a "weak local semicircle law" is valid and in the case of sparse random matrices this is provided by a result of Ref. 9 .

The universality of a single gap was established in Ref. 17 for Wigner matrices, i.e., for $p \sim O(1)$. The work in Ref. 26 also yields gap universality for DBM after the optimal time $t=N^{\epsilon-1}$ and so our task is similar to the proof of the correlation function universality in that we must establish step (3) and compare the gap distributions. However, the completion of step (3) presents a major difficulty. Previously, for gap universality, this step was based on results of Refs. 32 and 33 or Ref. 24 which states that the gap distribution of two Wigner ensembles coincide provided that the first four moments of these two ensembles match. However, these results were based on two inputs: first, certain level repulsion estimates; and second, an optimal eigenvalue rigidity estimate.

Optimal eigenvalue rigidity estimates for Wigner ensembles were proven in Refs. 18 and 20. This estimate states that for any eigenvalue $\lambda_{i}$ in the bulk, we have that $\left|\lambda_{i}-\gamma_{i}\right| \leq N^{-1+\delta}$ with overwhelming probability, where $\gamma_{i}$ is the deterministic classical location of the $i$ th eigenvalue. The best known rigidity result for sparse random matrices is from Ref. 9, where it was shown that the bulk eigenvalues satisfy $\left|\lambda_{i}-\gamma_{i}\right| \leq p^{-1} N^{-1+\delta}$ with overwhelming probability.

Moreover, we do not expect that optimal rigidity holds for sparse random matrices. In fact in Ref. 31, it was shown that for sparse random matrices, the linear statistics

$$
N^{-1} \sum_{i=1}^{N} \phi\left(\lambda_{i}\right) \rightarrow \mathcal{N}
$$

converges to a normal random variable with variance $O\left(\left(N^{2} p\right)^{-1}\right)$, for $\phi$ satisfying some regularity conditions. This implies that the fluctuations of the eigenvalues are at least of order $O\left((N \sqrt{p})^{-1}\right)$ on average, and so we do not expect optimal rigidity to hold if $p \ll 1$.

As mentioned above, the lack of rigidity for sparse ensembles resulted in the longer time to equilibrium for DBM being found in Ref. 8, and it again causes difficulty in trying to compare gap statistics. Rigidity results are a crucial input in establishing level repulsion estimates for Wigner matrices which are needed in order to compare the gap statistics of two ensembles. It was proven in Ref. 26 that a level repulsion estimate will hold for DBM after a short time. We show that one can combine the delocalization of eigenvectors together with the Ornstein-Uhlenbeck continuity lemma of Ref. 6 to pass this level repulsion from DBM to the initial sparse random matrix. This level repulsion estimate then gives us a key input for step (3) and we are able to conclude universality of the gap statistics.

Previous level repulsion estimates were obtained in Refs. 12 and 5 for Wigner ensembles whose entries have a smooth distribution. Estimates without a smoothness condition were obtained in Refs. 32 and 33 and also very recently in Ref. 29. A weak level repulsion estimate for Wigner matrices also follows from the results of Ref. 17.

In fact, our strategy outlined above applies to a wider class of random matrices than sparse or Wigner random matrices alone. We will prove that bulk universality holds for a class of random matrices obeying only a weak estimate on the distribution of its eigenvalues, a weak decay condition on the third moment of the entries, and an eigenvector delocalization estimate.

The remainder of the paper is outlined as follows. In Section II, we introduce the random matrix models under consideration, which we will call "stable" random matrices, and state our main results. In Section III, we obtain bulk universality for Gaussian divisible ensembles. In Section IV, we state and prove our level repulsion results for stable random matrices. In Section V, we complete step (3) outlined above and compare the bulk statistics of a general stable random matrix and a Gaussian divisible ensemble. In Section VI, we prove that sparse random matrices are stable and conclude universality for sparse random matrices.

\section{DEFINITION OF MODEL AND MAIN RESULTS}

In our paper, we will only state and prove our results for real symmetric random matrix ensembles. All of our methods extend with only notational changes to complex Hermitian ensembles. 


\section{A. Sparse random matrices}

In this section, we introduce the class of sparse random matrices that we study. We follow the notations and definitions of Refs. 9 and 8. The motivating example is the Erdős-Rényi matrix whose entries are independent up to the constraint that the matrix is symmetric, and equal to 1 with probability $p$ and 0 with probability $1-p$. It is notationally convenient to replace the parameter $p$ with $q$ defined through

$$
q:=\sqrt{N p} .
$$

We allow $q$ to depend on $N$. We also rescale the matrix so that the bulk of its spectrum lies in an interval of order 1. For the Erdôs-Rényi matrix, we define $H$ to be the $N \times N$ symmetric matrix whose entries $h_{i j}$ are independent up to $h_{i j}=h_{j i}$ and each element is distributed according to

$$
h_{i j}=\frac{\gamma}{q} \begin{cases}1 & \text { with probability } \frac{q^{2}}{N} \\ 0 & \text { with probability } 1-\frac{q^{2}}{N}\end{cases}
$$

where we have defined

$$
\gamma:=\left(1-\frac{q^{2}}{N}\right)^{-1 / 2} .
$$

We further extract the mean of each entry and write

$$
H=B+\gamma q|\boldsymbol{e}\rangle\langle\boldsymbol{e}|,
$$

where $\boldsymbol{e}$ is the unit vector

$$
\boldsymbol{e}:=\frac{1}{\sqrt{N}}(1, \ldots, 1)^{T} .
$$

Note that the matrix elements of $B$ are centered. It is easy to check that the matrix elements of $B$ satisfy the moment bounds

$$
\mathbb{E}\left[b_{i j}^{2}\right]=\frac{1}{N}, \quad \mathbb{E}\left[\left|b_{i j}\right|^{k}\right] \leq \frac{1}{N q^{k-2}}, \quad k \geq 2 .
$$

We are prompted to make the following definition. We introduce two parameters $q$ and $f$ which may be $N$-dependent.

Definition 2.1 (Sparse random matrices). $H$ is a sparse random matrix with sparsity parameter $q$ and mean $f$ if it is of the form

$$
H=B+f|\boldsymbol{e}\rangle\langle\boldsymbol{e}|,
$$

where $f$ is a deterministic number satisfying

$$
0 \leq f \leq N^{1 / 2}
$$

and $B$ is a matrix with real and independent entries up to the symmetry constraint $b_{i j}=b_{j i}$ which satisfy

$$
\mathbb{E}\left[b_{i j}\right]=0, \quad \mathbb{E}\left[\left|b_{i j}\right|^{2}\right]=\frac{1}{N}, \quad \mathbb{E}\left[\left|b_{i j}\right|^{k}\right] \leq \frac{C^{k}}{N q^{k-2}}
$$

for $1 \leq i \leq j \leq N$ and $2 \leq k \leq \log (N)^{10 \log \log N}$, where $C$ is a positive constant. We assume that $q$ satisfies

$$
N^{\alpha} \leq q \leq N^{1 / 2}
$$

for some $\alpha>0$. 


\section{B. Universality of sparse random matrices}

Our main result is the bulk universality of sparse random matrices as defined above.

Theorem 2.2. Let $H$ be a sparse random matrix as defined in Definition 2.1, with sparsity parameter q satisfying

$$
N^{\alpha} \leq q \leq N^{1 / 2}
$$

for some number $\alpha>0$. Then, $H$ exhibits bulk universality in the following two forms. First, $H$ has the single gap universality in the bulk. For any $\kappa>0$ and index $i \in[[\kappa N,(1-\kappa) N]]$,

$$
\begin{aligned}
& \lim _{N \rightarrow \infty} \mathbb{E}^{(H)}\left[O\left(N \rho_{s c}\left(\gamma_{i}\right)\left(\lambda_{i}-\lambda_{i+1}\right), \ldots, N \rho_{s c}\left(\gamma_{i}\right)\left(\lambda_{i}-\lambda_{i+n}\right)\right)\right] \\
& \quad-\mathbb{E}^{(G O E)}\left[O\left(N \rho_{s c}\left(\gamma_{i}\right)\left(\lambda_{i}-\lambda_{i+1}\right), \ldots, N \rho_{s c}\left(\gamma_{i}\right)\left(\lambda_{i}-\lambda_{i+n}\right)\right)\right]=0 .
\end{aligned}
$$

Second, the averaged n-point correlation functions of $H$ are universal in the bulk. We denote the n-point correlation function functions of $H$ and GOE by $\rho_{H}^{(n)}$ and $\rho_{G O E}^{(n)}$, respectively, then for any $\delta>0$ and $E \in(-2,2)$, and $b \geq N^{-1+\delta}$,

$$
\begin{aligned}
\lim _{N \rightarrow \infty} & \int_{E-b}^{E+b} \int_{\mathbb{R}^{n}} O\left(\alpha_{1}, \ldots, \alpha_{n}\right)\left\{\frac{1}{\rho_{s c}(E)^{n}} \rho_{H}^{(n)}\left(E^{\prime}+\frac{\alpha_{1}}{N \rho_{s c}(E)}, \ldots, E^{\prime}+\frac{\alpha_{n}}{N \rho_{s c}(E)}\right)\right. \\
& \left.-\frac{1}{\rho_{s c}(E)^{n}} \rho_{G O E}^{(n)}\left(E^{\prime}+\frac{\alpha_{1}}{N \rho_{s c}(E)}, \ldots, E^{\prime}+\frac{\alpha_{n}}{N \rho_{s c}(E)}\right)\right\} \mathrm{d} \alpha_{1} \ldots \mathrm{d} \alpha_{n} \frac{\mathrm{d} E^{\prime}}{2 b}=0,
\end{aligned}
$$

where the test function $O \in C_{c}^{\infty}\left(\mathbb{R}^{n}\right)$.

\section{Stable random matrices}

While our main goal is to study the sparse random matrices defined above, we note that our analysis applies to a somewhat more general class of random matrices. We consider an $N \times N$ real symmetric random matrix $H=\left(h_{i j}\right)_{1 \leq i, j \leq N}$, which satisfy $\mathbb{E}\left[h_{i j}\right]=f$ and $\mathbb{E}\left[\left(h_{i j}-f\right)^{2}\right]=s_{i j}$. We assume that there are constants $c_{1}$ and $c_{2}$ such that

$$
c_{1} N^{-1} \leq s_{i j} \leq c_{2} N^{-1},
$$

and the mean $0 \leq f \leq N^{C}$ may depend on $N$.

We define the following matrix stochastic differential equation (Ref. 6) which is an OrnsteinUhlenbeck version of the Dyson Brownian motion. The dynamics of the matrix entries are given by the stochastic differential equations

$$
\mathrm{d}\left(h_{i j}(t)-f\right)=\frac{\mathrm{d} B_{i j}(t)}{\sqrt{N}}-\frac{1}{2 N s_{i j}}\left(h_{i j}(t)-f\right) \mathrm{d} t,
$$

where $B$ is symmetric with $\left(B_{i j}(t)\right)_{1 \leq i \leq j \leq N}$ a family of independent Brownian motions. We denote $H_{t}=\left(h_{i j}(t)\right)_{1 \leq i, j \leq N}$, and so, $H_{0}=H$ is our original matrix. More explicitly, for the entries of $H_{t}$, we have

$$
h_{i j}(t)=f+\mathrm{e}^{-\frac{t}{2 N s_{i j}}}\left(h_{i j}(0)-f\right)+\frac{1}{\sqrt{N}} \int_{0}^{t} \mathrm{e}^{\frac{s-t}{2 N s_{i j}}} \mathrm{~d} B_{i j}(s) .
$$

Clearly, for any $t \geq 0$, we have $\mathbb{E}\left[h_{i j}(t)\right]=f$ and $\mathbb{E}\left[\left(h_{i j}(t)-f\right)^{2}\right]=s_{i j}$. More importantly, the law of $h_{i j}(t)$ is Gaussian divisible, i.e., it contains a copy of Gaussian random variable with variance $O\left(t N^{-1}\right)$. Therefore, $H_{t}$ can be written as

$$
H_{t} \stackrel{d}{=} H_{t}^{(1)}+\sqrt{\frac{r\left(1-e^{-t / r}\right)}{2}} G
$$

where $r=\min _{i \leq j}\left\{N s_{i j}\right\}, G$ denotes a standard gaussian orthogonal ensemble, which is independent of $H_{t}^{(1)}$. The entries of the matrix $H_{t}^{(1)}$ are given by 


$$
\left(H_{t}^{(1)}\right)_{i j} \stackrel{d}{=} f+\mathrm{e}^{-\frac{t}{2 N s_{i j}}}\left(h_{i j}(0)-f\right)+\sqrt{N s_{i j}\left(1-e^{-\frac{t}{N s_{i j}}}\right)-\frac{1+\delta_{i j}}{2} r\left(1-e^{-\frac{t}{r}}\right)} \frac{\tilde{B}_{i j}(t)}{\sqrt{N}},
$$

where $\tilde{B}$ is symmetric with $\left(\tilde{B}_{i j}(t)\right)_{1 \leq i \leq j \leq N}$ a family of independent Brownian motions.

We define the deformed matrix $\theta^{a b} H_{t}$ by

$$
\left(\theta^{a b} H_{t}\right)_{k l}:=f+\theta_{k l}^{a b}\left(h_{k l}(t)-f\right),
$$

where $\theta_{k l}^{a b}=1$ unless $\{k, l\}=\{a, b\}$ in which case $\theta_{a b}^{a b}=\theta_{b a}^{a b}$ will be a number satisfying $0 \leq \theta_{a b}^{a b}=$ $\theta_{b a}^{a b} \leq 1$.

Definition 2.3. Let $A$ be an $N \times N$ deterministic real symmetric matrix. We denote the eigenvalues of $A$ as $\left\{\lambda_{1}, \lambda_{2}, \ldots, \lambda_{N}\right\}$ and corresponding eigenvectors $\left\{u_{1}, u_{2}, \ldots, u_{N}\right\}$. For any (small) number $\delta>0$, we call the matrix $M \delta$-general if:

(1) The eigenvectors of $A$ are completely delocalized: $\sup _{i, j}\left|u_{i}(j)\right|^{2} \leq C N^{-1+\delta}$.

(2) The eigenvalues of $A$ do not accumulate: there is an universal constant $C$, such that for any interval $I$ with length $|I| \geq N^{-1+\delta}$, we have \#\{i: $\left.\lambda_{i} \in I\right\} \leq C|I| N$.

Definition 2.4. We call the random matrix $H$ stable if:

(1) The entries of $H$ are independent up to symmetry.

(2) For any time $t=N^{-1+\epsilon}$, where $\epsilon>0$ can be arbitrarily small, the random matrix $H_{t}^{(1)}$ defined in (2.17) satisfies the weak local semicircle law, i.e., for any (large) number $D>0$, and (small) number $\delta>0$, the following holds with probability larger than $1-N^{-D}$ :

$$
\left|\frac{1}{N} \operatorname{Tr}\left(H_{t}^{(1)}-E-i \eta\right)^{-1}-m_{s c}(E+i \eta)\right| \leq N^{-c(\delta)},
$$

uniformly for $-5 \leq E \leq 5, N^{-1+\delta} \leq \eta \leq 10$, where $c(\delta)>0$ is some constant depending on $\delta$, and $\operatorname{spec}\left(H_{t}^{(1)}\right) \subseteq\left(-3, N^{C}\right)$ for some fixed $C$.

(3) The first two moments of entries of $H$ satisfy $\mathbb{E}\left[h_{i j}\right]=f$ and $\mathbb{E}\left[\left(h_{i j}-f\right)^{2}\right]=s_{i j}$. We assume that there are constants $c_{1}$ and $c_{2}$ such that $c_{1} N^{-1} \leq s_{i j} \leq c_{2} N^{-1}$, and the mean $0 \leq f \leq N^{C}$ may depend on $N$. For the third moment, there exists some universal constant $\alpha$, such that $\mathbb{E}\left[\left|h_{i j}-f\right|^{3}\right] \leq N^{-1-\alpha}$.

(4) For any time $0 \leq s \leq N^{-1+\epsilon}$, where $\epsilon>0$ can be arbitrarily small, indexes $0 \leq a, b \leq N$, any (large) number $D>0$, and (small) number $\delta>0$, with probability larger than $1-N^{-D}, \theta^{a b} H_{s}$ is $\delta$-general for any choice of $\theta_{a b}^{a b} \in[0,1]$, with the constants in Definition 2.3 uniformly in $s$.

Remark 2.5. Second condition (2) implies that for any $\kappa>0$, the eigenvalues $\lambda_{i}\left(H_{t}^{(1)}\right) \in(-2+$ $\left.\kappa^{2 / 3}, 2-\kappa^{2 / 3}\right)$ for $i \in[[2 \kappa N,(1-2 \kappa) N]]$ with overwhelming probability.

Remark 2.6. In order to simplify our proof, we have assumed that the matrix elements are independent. Independence is mainly used in the comparison Lemma 4.3, which will still hold if the matrix entries of $H$ are weakly correlated.

Remark 2.7. The motivating example of our paper is the sparse random matrix, and we have therefore assumed that the Stieltjes transform of the empirical eigenvalue distribution of $H_{t}^{(1)}$ is close to $m_{s c}$, i.e., the semicircle law. The semicircle law, however, does not play an active role and our methods can be applied to the case in which the semicircle law is replaced by other densities. We will not pursue this direction and refer the interested reader to Refs. 2, 3, and 1 for examples in which the limiting eigenvalue density differs from the semicircle law.

In this paper, we will prove that the local statistics of stable random matrices are universal.

Theorem 2.8. Let $H$ be a stable random matrix as defined in Definition 2.4. The local statistics of $H$ in the bulk are universal. First, $H$ has gap universality with a fixed label in the bulk. For any 
$\kappa>0$ and index $i \in[[\kappa N,(1-\kappa) N]]$, we have

$$
\begin{aligned}
\lim _{N \rightarrow \infty} & \mathbb{E}^{(H)}\left[O\left(N \rho_{s c}\left(\gamma_{i}\right)\left(\lambda_{i}-\lambda_{i+1}\right), \ldots, N \rho_{s c}\left(\gamma_{i}\right)\left(\lambda_{i}-\lambda_{i+n}\right)\right)\right] \\
& -\mathbb{E}^{(G O E)}\left[O\left(N \rho_{s c}\left(\gamma_{i}\right)\left(\lambda_{i}-\lambda_{i+1}\right), \ldots, N \rho_{s c}\left(\gamma_{i}\right)\left(\lambda_{i}-\lambda_{i+n}\right)\right)\right]=0 .
\end{aligned}
$$

The averaged n-point correlation functions of $H$ are universal in the bulk. We denote the n-point correlation function functions of $H$ and GOE by $\rho_{H}^{(n)}$ and $\rho_{G O E}^{(n)}$, respectively, then for any $\delta>0$ and $E \in(-2,2)$, and $b \geq N^{-1+\delta}$

$$
\begin{aligned}
\lim _{N \rightarrow \infty} & \int_{E-b}^{E+b} \int_{\mathbb{R}^{n}} O\left(\alpha_{1}, \ldots, \alpha_{n}\right)\left\{\frac{1}{\rho_{s c}(E)^{n}} \rho_{H}^{(n)}\left(E^{\prime}+\frac{\alpha_{1}}{N \rho_{s c}(E)}, \ldots, E^{\prime}+\frac{\alpha_{n}}{N \rho_{s c}(E)}\right)\right. \\
& \left.-\frac{1}{\rho_{s c}(E)^{n}} \rho_{G O E}^{(n)}\left(E^{\prime}+\frac{\alpha_{1}}{N \rho_{s c}(E)}, \ldots, E^{\prime}+\frac{\alpha_{n}}{N \rho_{s c}(E)}\right)\right\} \mathrm{d} \alpha_{1} \ldots \mathrm{d} \alpha_{n} \frac{d E^{\prime}}{2 b}=0 .
\end{aligned}
$$

Above, the observable $O \in C_{c}^{\infty}\left(\mathbb{R}^{n}\right)$.

\section{BULK UNIVERSALITY OF $\boldsymbol{H}_{t}$}

The goal of this section is to establish bulk universality for the matrix valued stochastic process $H_{t}$ defined as in (2.15) after a short time $t=N^{-1+\epsilon}$.

Theorem 3.1. Let $H$ be a stable random matrix, and let $H_{t}$ be defined as in (2.15). For any small $\epsilon, \kappa>0$ there is a constant $c>0$, which depends on $\epsilon$, such that the following holds for $t=N^{-1+\epsilon}$ and any index $i \in[[\kappa N,(1-\kappa) N]]$ :

$$
\begin{aligned}
& \mid \mathbb{E}^{\left(H_{t}\right)}\left[O\left(N\left(\lambda_{i}-\lambda_{i+1}\right), \ldots, N\left(\lambda_{i}-\lambda_{i+n}\right)\right)\right] \\
- & \mathbb{E}^{(G O E)}\left[O\left(N\left(\lambda_{i}-\lambda_{i+1}\right), \ldots, N\left(\lambda_{i}-\lambda_{i+n}\right)\right)\right] \mid \leq N^{-c} .
\end{aligned}
$$

Moreover, for any $\delta, \kappa>0, E \in(-2+\kappa, 2-\kappa)$ and $b \geq N^{-1+\delta}$, we have

$$
\begin{aligned}
& \mid \int_{E-b}^{E+b} \int_{\mathbb{R}^{n}} O\left(\alpha_{1}, \ldots, \alpha_{n}\right)\left\{\frac{1}{\rho_{\mathrm{sc}}(E)^{n}} \rho_{H_{t}}^{(n)}\left(E^{\prime}+\frac{\alpha_{1}}{N \rho_{\mathrm{sc}}(E)}, \ldots, E^{\prime}+\frac{\alpha_{n}}{N \rho_{\mathrm{sc}}(E)}\right)\right. \\
& \left.-\frac{1}{\rho_{\mathrm{sc}}(E)^{n}} \rho_{G O E}^{(n)}\left(E^{\prime}+\frac{\alpha_{1}}{N \rho_{\mathrm{sc}}(E)}, \ldots, E^{\prime}+\frac{\alpha_{n}}{N \rho_{\mathrm{sc}}(E)}\right)\right\} \mathrm{d} \alpha_{1} \ldots \mathrm{d} \alpha_{n} \frac{\mathrm{d} E^{\prime}}{b} \mid \leq N^{-c},
\end{aligned}
$$

where the test function $O \in C_{c}^{\infty}\left(\mathbb{R}^{n}\right)$.

For the proof, we shall first restate the main result of Ref. 26 in a form convenient for our proof. For this we will introduce some notation. For a deterministic matrix $A$, we define

$$
A_{t}:=A+\vartheta_{t} G, \quad \vartheta_{t}:=\sqrt{\frac{r\left(1-e^{-t / r}\right)}{2}}=O\left(t^{\frac{1}{2}}\right),
$$

where $G$ is a Gaussian orthogonal ensemble matrix and $r$ is the constant from (2.17). We denote Stieltjes transform of the free convolution of the empirical eigenvalue distribution of $A$ and $\theta G$, and so, $m_{0}$ is the Stieltjes transform of empirical eigenvalue distribution of $A$. More explicitly, it is given by

$$
m_{t}(z):=m_{0}\left(z+\vartheta_{t}^{2} m_{t}(z)\right)=\frac{1}{N} \sum_{i=1}^{N} \frac{1}{\lambda_{i}(A)-z-\vartheta_{t}^{2} m_{t}(z)} .
$$

The free convolution is well-studied, see, e.g., Ref. 4. It is known that $m_{t}$ is the Stieltjes transform of a measure with a density which we denote by $\rho_{t}$ which is analytic on the interior of its support, for any $t>0$. Denote the classical eigenvalue locations of the density $\rho_{s c}$ and $\rho_{t}$ by $\gamma_{i}$ and $\gamma_{i, t}$, respectively,

$$
\int_{-\infty}^{\gamma_{i}} \rho_{s c}(x) d x=\frac{i}{N}, \quad \int_{-\infty}^{\gamma_{i, t}} \rho_{t}(x) d x=\frac{i}{N}
$$

The following follows from Ref. 26 (Theorem 2.5). 
Theorem 3.2. Suppose that there are constants $c_{1}>0$ and $C_{1}>0$ such that

$$
c_{1} \leq \operatorname{Im}\left[m_{0}(E+\mathrm{i} \eta)\right] \leq C_{1}
$$

for all $E \in(-2+\kappa, 2-\kappa)$ and $N^{-1+\epsilon / 3} \leq \eta \leq 10$. Suppose furthermore that $\operatorname{spec}(A) \subseteq\left[-C, N^{C}\right)$ for some fixed $C$. Let $i$ be such that $\lambda_{i}(A) \in(-2+2 \kappa, 2-2 \kappa)$. Then for any $\epsilon>0, t=N^{-1+\epsilon}$, there exists a small constant $c>0$, which depends on $\epsilon$, such that for indexes $i_{1}, \ldots, i_{n} \in \mathbb{N}$ and $i_{k} \leq N^{c}$,

$$
\begin{aligned}
& \mid \mathbb{E}^{\left(A_{t}\right)}\left[O\left(\rho_{t}\left(\gamma_{i, t}\right) N\left(\lambda_{i}-\lambda_{i+i_{1}}\right), \ldots, \rho_{t}\left(\gamma_{i, t}\right) N\left(\lambda_{i}-\lambda_{i+i_{n}}\right)\right)\right] \\
- & \mathbb{E}^{(G O E)}\left[O\left(\rho_{\mathrm{sc}}\left(\gamma_{j}\right) N\left(\lambda_{j}-\lambda_{j+i_{1}}\right), \ldots, \rho_{\mathrm{sc}}\left(\gamma_{j}\right) N\left(\lambda_{j}-\lambda_{j+i_{n}}\right)\right)\right] \mid \leq N^{-c}
\end{aligned}
$$

for all large enough $N \geq N(\epsilon)$. Above $j$ is any index satisfying $j \in\left[\left[\kappa_{1} N,\left(1-\kappa_{1}\right) N\right]\right]$, where $\kappa_{1}>0$.

We will apply the above theorem by conditioning on $H_{t}^{(1)}$ and taking $A=H_{t}^{(1)}$. However, we must take care of the scaling by $\rho_{t}\left(\gamma_{i, t}\right)$ in the above theorem statement. This becomes a random variable depending on $H_{t}^{(1)}$. We want to replace it by the deterministic quantity $\rho_{\mathrm{sc}}\left(\gamma_{i}\right)$; we will see that our assumption on the weak local law of $H_{t}^{(1)}$ combined with continuity of the free convolution allows us to do this.

Define the spectral domain $\mathcal{F}=\left\{z=E+i \eta: E \in(-5,5), N^{-1+\epsilon / 3} \leq \eta \leq 10\right\}$, and the set of real symmetric $N \times N$ matrices

$$
\mathcal{D}:=\left\{A:\left|m_{0}(z)-m_{\mathrm{sc}}(z)\right| \leq N^{-\omega}, z \in \mathcal{F}\right\} \cap\left\{\operatorname{spec}(A) \subseteq\left(-3, N^{C}\right)\right\},
$$

where $\omega$ satisfies $0<\omega \leq \min \{\epsilon / 3, c(\epsilon / 3),(1-\epsilon) / 4\}$, and $c(\epsilon / 3)$ is from the constant in (2.19) of Definition 2.4.

Lemma 3.3. Let $\kappa, \omega>0$ and $A \in \mathcal{D}$ as above. Then, for any $\epsilon>0$ and $t=N^{-1+\epsilon}$,

$$
\left|m_{t}(z)-m_{\mathrm{sc}}(z)\right| \leq C N^{-\omega},
$$

uniformly for any $z \in\left\{z=E+\mathrm{i} \eta: E \in(-4,4), N^{-1+\epsilon / 3} \leq \eta \leq 9\right\}$.

Proof. From Jensen's inequality, we have

$$
\left|m_{t}\right|^{2} \leq \frac{1}{N} \sum \frac{1}{\left|\lambda_{i}(A)-z-\vartheta_{t}^{2} m_{t}(z)\right|^{2}}=\frac{\operatorname{Im}\left[m_{t}(z)\right]}{\operatorname{Im}\left[z+\vartheta_{t}^{2} m_{t}(z)\right]} \leq \vartheta_{t}^{-2} .
$$

Therefore, we have $\left|\vartheta_{t}^{2} m_{t}\right| \leq \vartheta_{t}=O\left(t^{1 / 2}\right)$. Since $A \in \mathcal{D}$, for any $z=E+i \eta$, such that $E \in(-4,4)$ and $N^{-1+\epsilon / 3} \leq \eta \leq 9$, we have that $z+\vartheta_{t}^{2} m_{t} \in \mathcal{F}$. Defining relation (3.7) leads to

$$
m_{t}(z)=m_{0}\left(z+\vartheta_{t}^{2} m_{t}(z)\right)=m_{s c}\left(z+\vartheta_{t}^{2} m_{t}(z)\right)+O\left(N^{-\omega}\right) .
$$

To control $m_{s c}\left(z+\vartheta_{t}^{2} m_{t}\right)$, we have the following stability estimate of $m_{s c}$ : for any $z, \Delta z$ with non-negative imaginary part, and $|\Delta z| \leq 1$, then

$$
\left|m_{s c}(z+\Delta z)-m_{s c}(z)\right| \leq 2|\Delta z|^{1 / 2} .
$$

Therefore, (3.9) gives us

$$
\left|m_{t}(z)-m_{s c}(z)\right| \leq\left|m_{t}(z)-m_{s c}\left(z+\vartheta_{t}^{2} m_{t}\right)\right|+\left|m_{s c}\left(z+\vartheta_{t}^{2} m_{t}\right)-m_{s c}(z)\right| \leq C N^{-\omega},
$$

given that $\omega<(1-\epsilon) / 4$.

From this lemma, we conclude the following.

Lemma 3.4. For any $\epsilon, \kappa>0$, time $t=N^{-1+\epsilon}$, any real symmetric matrix $A \in \mathcal{D}$, and $E \in$ $(-2+\kappa, 2-\kappa)$, we have

$$
\left|\rho_{t}(E)-\rho_{\mathrm{sc}}(E)\right| \leq C N^{-\omega} .
$$

Moreover, for any index $i$ such that $\lambda_{i}(A) \in(-2+\kappa, 2-\kappa)$, we have

$$
\left|\rho_{t}\left(\gamma_{i, t}\right)-\rho_{\mathrm{sc}}\left(\gamma_{i}\right)\right| \leq C N^{-\omega / 2},
$$

where the constant $\omega$ is from definition (3.7) of the set $\mathcal{D}$. 
Proof. From Ref. 26 (Lemma 7.1), the derivative of $\rho_{t}$ satisfies $\left|\rho_{t}^{\prime}(E)\right| \leq C / t$ for $E \in(-2+$ $\kappa, 2-\kappa)$, where we use the fact $\vartheta_{t}^{2}=O(t)$. Moreover, the same lemma also shows that $\rho_{t}(E) \leq C$ on $(-2+\kappa / 2,2-\kappa / 2)$. Given $E \in(-2+\kappa, 2-\kappa)$, we denote the intervals $I_{1}=\left[E-N^{-1+2 \epsilon / 3}, E+\right.$ $\left.N^{-1+2 \epsilon / 3}\right], I_{2}=\left\{x: N^{-1+2 \epsilon / 3}<|x-E|<\kappa / 2\right\}$, and $I_{3}=\mathbb{R} \backslash\left(I_{1} \cup I_{2}\right)$. We take $\eta=N^{-1+\epsilon / 3}$, then

$$
\begin{aligned}
& \left|\rho_{t}(E)-\frac{1}{\pi} \operatorname{Im}\left[m_{t}(E+i \eta)\right]\right| \\
\leq & \left|\frac{1}{\pi} \int_{I_{1}} \frac{\left(\rho_{t}(E)-\rho_{t}(x)\right) \eta \mathrm{d} x}{(x-E)^{2}+\eta^{2}} d x\right|+\left|\frac{1}{\pi} \int_{I_{2} \cup I_{3}} \frac{\rho_{t}(E) \eta \mathrm{d} x}{(x-E)^{2}+\eta^{2}}\right| \\
+ & \left|\frac{1}{\pi} \int_{I_{2}} \frac{\rho_{t}(x) \eta \mathrm{d} x}{(x-E)^{2}+\eta^{2}}\right|+\left|\frac{1}{\pi} \int_{I_{3}} \frac{\rho_{t}(x) \eta \mathrm{d} x}{(x-E)^{2}+\eta^{2}}\right| \\
\leq & \sup _{x \in I_{1}}\left|\rho_{t}(x)-\rho_{t}(E)\right|+C N^{-\epsilon / 3}+C N^{-\epsilon / 3}+C \frac{\eta}{\kappa^{2}} \int_{I_{3}} \rho_{t}(x) \mathrm{d} x \leq C N^{-\epsilon / 3},
\end{aligned}
$$

where we have used $\left|\rho_{t}(x)\right| \leq C$ on $I_{1} \cup I_{2}$. Moreover, we have

$$
\begin{aligned}
& \left|\frac{1}{\pi} \operatorname{Im}\left[m_{t}(E+i \eta)\right]-\rho_{s c}(E)\right| \\
\leq & \frac{1}{\pi}\left|\operatorname{Im}\left[m_{t}(E+i \eta)\right]-\operatorname{Im}\left[m_{s c}(E+i \eta)\right]\right|+\frac{1}{\pi}\left|\operatorname{Im}\left[m_{s c}(E+i \eta)\right]-\frac{1}{\pi} \operatorname{Im}\left[m_{s c}(E)\right]\right| \\
\leq & N^{-\omega}+\frac{2}{\pi} \sqrt{\eta} \leq C N^{-\omega},
\end{aligned}
$$

given $\omega \leq(1-\epsilon) / 2$. (3.13) and (3.14) together lead to (3.11).

Moreover, by our hypothesis that $A \in \mathcal{D}$, $\operatorname{spec}(A)$ is bounded below by 3 . Therefore, the density $\rho_{t}$ is also bounded below: supp $\rho_{t} \in[-4, \infty)$. Therefore, if $i$ is such that $\lambda_{i}(A) \in(-2+\kappa, 2-\kappa)$ then

$$
\left|\gamma_{i, t}-\gamma_{i}\right| \leq N^{-\omega / 2}
$$

This follows from Ref. 26 (Lemma 7.17). Therefore,

$$
\left|\rho_{t}\left(\gamma_{i, t}\right)-\rho_{\mathrm{sc}}\left(\gamma_{i}\right)\right| \leq\left|\rho_{t}\left(\gamma_{i, t}\right)-\rho_{\mathrm{sc}}\left(\gamma_{i, t}\right)\right|+\left|\rho_{\mathrm{sc}}\left(\gamma_{i, t}\right)-\rho_{\mathrm{sc}}\left(\gamma_{i}\right)\right| \leq C N^{-\omega / 2} .
$$

\section{A. Proof of Theorem 3.1}

By the hypotheses of stability of $H$, we have that $H_{t}^{(1)} \in \mathcal{D}$ with probability greater than $1-N^{-D}$ for any large $D$. We denote the density of free convolution of $H_{t}^{(1)}$ and $\vartheta_{t} G$ as $\rho_{t}^{H}$, and its $i$ th classical eigenvalue location as $\gamma_{i, t}^{H}$. From Theorem 3.2, we have

$$
\begin{gathered}
\mid \mathbb{E}^{\left(H_{t}\right)}\left[O\left(\rho_{t}^{H}\left(\gamma_{i, t}^{H}\right) N\left(\lambda_{i}-\lambda_{i+i_{1}}\right), \ldots, \rho_{t}^{H}\left(\gamma_{i, t}^{H}\right) N\left(\lambda_{i}-\lambda_{i+i_{n}}\right)\right) \mid H_{t}^{(1)}\right] \\
-\mathbb{E}^{(G O E)}\left[O\left(\rho_{\mathrm{sc}}\left(\gamma_{i}\right) N\left(\lambda_{i}-\lambda_{i+i_{1}}\right), \ldots, \rho_{\mathrm{sc}}\left(\gamma_{i}\right) N\left(\lambda_{i}-\lambda_{i+i_{n}}\right)\right)\right] \mid \leq N^{-c} .
\end{gathered}
$$

But then by Lemma 3.4, we have that $\left|\rho_{t}^{H}\left(\gamma_{i, t}^{H}\right)-\rho_{s c}\left(\gamma_{i}\right)\right| \leq N^{-\omega / 2}$, therefore,

$$
\begin{aligned}
& \mid \mathbb{E}^{\left(H_{t}\right)}\left[O\left(\rho_{t}^{H}\left(\gamma_{i, t}^{H}\right) N\left(\lambda_{i}-\lambda_{i+i_{1}}\right), \ldots, \rho_{t}^{H}\left(\gamma_{i, t}^{H}\right) N\left(\lambda_{i}-\lambda_{i+i_{n}}\right)\right) \mid H_{t}^{(1)}\right] \\
- & \mathbb{E}^{\left(H_{t}\right)}\left[O\left(\rho_{\mathrm{sc}}\left(\gamma_{i}\right) N\left(\lambda_{i}-\lambda_{i+i_{1}}\right), \ldots, \rho_{\mathrm{sc}}\left(\gamma_{i}\right) N\left(\lambda_{i}-\lambda_{i+i_{n}}\right)\right) \mid H_{t}^{(1)}\right] \mid \leq C N^{-\omega / 2}
\end{aligned}
$$

for some $C>0$ depending on first derivative and the support of the test function $O$. We therefore obtain that for $H_{t}^{(1)} \in \mathcal{D}$ that

$$
\begin{aligned}
& \mid \mathbb{E}^{\left(H_{t}\right)}\left[O\left(\rho_{\mathrm{sc}}\left(\gamma_{i}\right) N\left(\lambda_{i}-\lambda_{i+i_{1}}\right), \ldots, \rho_{\mathrm{sc}}\left(\gamma_{i}\right) N\left(\lambda_{i}-\lambda_{i+i_{n}}\right)\right) \mid H_{t}^{(1)}\right] \\
- & \mathbb{E}^{(G O E)}\left[O\left(\rho_{\mathrm{sc}}\left(\gamma_{i}\right) N\left(\lambda_{i}-\lambda_{i+i_{1}}\right), \ldots, \rho_{\mathrm{sc}}\left(\gamma_{i}\right) N\left(\lambda_{i}-\lambda_{i+i_{n}}\right)\right)\right] \mid \leq N^{-c}
\end{aligned}
$$

if we choose $c$ small enough, such that $c<\omega / 2$. And if we take expectation over $H_{t}^{(1)}$, (3.1) follows. 
Now let $\tilde{\rho}_{t}^{(n)}$ be the $n$-point correlation functions of $H_{t}$ conditioned on $H_{t}^{(1)}$. It is well known that estimate (3.17) together with the optimal rigidity of the eigenvalues of $H_{t}$ (Ref. 26, Theorem 3.3) implies that for $H_{t}^{(1)} \in \mathcal{D}$, we have

$$
\begin{gathered}
\mid \int_{E-b}^{E+b} \int_{\mathbb{R}^{n}} O\left(\alpha_{1}, \ldots, \alpha_{n}\right)\left\{\frac{1}{\rho_{\mathrm{sc}}(E)^{n}} \tilde{\rho}_{t}^{(n)}\left(E^{\prime}+\frac{\alpha_{1}}{N \rho_{\mathrm{sc}}(E)}, \ldots, E^{\prime}+\frac{\alpha_{n}}{N \rho_{\mathrm{sc}}(E)}\right)\right. \\
\left.-\frac{1}{\rho_{\mathrm{sc}}(E)^{n}} \rho_{G O E}^{(n)}\left(E^{\prime}+\frac{\alpha_{1}}{N \rho_{\mathrm{sc}}(E)}, \ldots, E^{\prime}+\frac{\alpha_{n}}{N \rho_{\mathrm{sc}}(E)}\right)\right\} \mathrm{d} \alpha_{1} \ldots \mathrm{d} \alpha_{n} \frac{\mathrm{d} E^{\prime}}{b} \mid \leq N^{-c}
\end{gathered}
$$

for $b=N^{-1+\delta}$ for any $\delta>0$. For this argument, we refer the reader to, e.g., Ref. 13 (Theorem 2.1). We conclude (3.2) by integrating over $H_{t}^{(1)}$.

\section{LEVEL REPULSION FOR STABLE RANDOM MATRICES}

In this section, we prove the following level repulsion estimate for stable random matrices. It will be used for the comparison of the single gap statistics between $H$ and $H_{t}$ in Section V.

Theorem 4.1. Let $H$ be a stable random matrix as defined in Section II C. Given any $0<\tau<$ $\alpha / 8$, any (small) number $\kappa>0$, and any index $i \in[[\kappa N,(1-\kappa) N]]$, we have

$$
\mathbb{P}\left(\left|\lambda_{i}(H)-\lambda_{i+1}(H)\right| \leq N^{-1-\tau}\right) \leq N^{-\tau / 2} .
$$

Remark 4.2. The above estimate suffices for the comparison of the single gap statistics of $H$ and $H_{t}$. We have not tried to optimize the exponent $-\tau / 2$, which is far from optimal. The proof below is easily modified to give $-\tau+\delta$ for any $\delta>0$.

It was proven in Ref. 26 that a level repulsion estimate holds for the matrix $H_{t}$ for $t=N^{-1+\epsilon}$. To obtain the level repulsion estimate for $H$, we need to prove that the change of eigenvalues up to time $t=N^{-1+\epsilon}$ is negligible. For this, we will repeatedly use the following lemma which asserts continuity of DBM when viewed as a matrix Ornstein-Uhlenbeck process. It is a minor modification of Ref. 6 (Lemma A.2).

Lemma 4.3. Let $H$ be an $N \times N$ real symmetric random matrix $H=\left(h_{i j}\right)_{1 \leq i, j \leq N}$, where the $h_{i j}$ 's are independent up to symmetry constraint $h_{i j}=h_{j i}$. Suppose that for constants $c_{1}, c_{2}, c_{3}$ its entries satisfy $\mathbb{E}\left[h_{i j}\right]=f$ and $\mathbb{E}\left[\left(h_{i j}-f\right)^{2}\right]=s_{i j}$, where $c_{1} N^{-1} \leq s_{i j} \leq c_{2} N^{-1}$ and $f \geq 0$ may depend on $N$.

Define $H_{t}$ as in (2.15). Let $F$ be a smooth function on the space of real symmetric matrices satisfying

$$
\sup _{0 \leq s \leq t, a \leq b} \mathbb{E}\left[\left(N^{2}\left|h_{a b}(s)-f\right|^{3}+N\left|h_{a b}(s)-f\right|\right) \sup _{\theta^{a b}}\left|\partial_{a b}^{(3)} F\left(\theta^{a b} H_{s}\right)\right| \leq B .\right.
$$

Above, the deformed matrix $\theta^{a b} H_{s}$ is defined by $\left(\theta^{a b} H\right)_{k l}=f+\theta_{k l}^{a b}\left(h_{k l}-f\right)$, where $\theta_{k l}^{a b}=1$ unless $\{k, l\}=\{a, b\}$ and $\theta_{a b}^{a b}=\theta_{b a}^{a b}$ is a number satisfying $0 \leq \theta_{a b}^{a b}=\theta_{b a}^{a b} \leq 1$. Then

$$
\left|\mathbb{E}\left[F\left(H_{t}\right)\right]-\mathbb{E}\left[F\left(H_{0}\right)\right]\right| \leq C t B .
$$

Given a real symmetric matrix $A$, we denote its eigenvalues by $\left\{\lambda_{1}, \lambda_{2}, \ldots, \lambda_{N}\right\}$, and corresponding eigenvectors $\left\{u_{1}, u_{2}, \ldots, u_{N}\right\}$. If $\lambda_{i}$ is a simple eigenvalue of $A$, we define $P_{i}$ to be the orthogonal projection to the one-dimensional eigenspace corresponding to $\lambda_{i}$, and the resolvent $R_{i}(A)$ the unique real symmetric matrix inverting $\lambda_{i}-A$ on the range of $I-P_{i}(A)$, and vanishing on the range of $P_{i}(A) . R_{i}(A)$ can be written explicitly as

$$
R_{i}(A)=\sum_{j: j \neq i} \frac{1}{\lambda_{i}-\lambda_{j}} u_{j} u_{j}^{*}
$$


Moreover, $R_{i}(A)$ can be written as the following contour integral:

$$
R_{i}(A)=\frac{1}{2 \pi i} \oint_{\left|z-\lambda_{i}\right|=\omega} \frac{1}{\lambda_{i}-z} \frac{1}{A-z} d z
$$

where we pick the contour $\omega$ to enclose only $\lambda_{i}$. From the above formula, it is clear that $R_{i}(A)$ is a smooth function on a neighbourhood of $A$ if $\lambda_{i}$ is a single eigenvalue. We refer the reader to the book Ref. 30 (Chapter XII) for related properties.

If $\lambda_{i}$ is a single eigenvalue of $A$, we define the quantity

$$
Q_{i}(A)=\frac{1}{N^{2}} \operatorname{Tr}\left(R_{i}(A)^{2}\right)=\frac{1}{N^{2}} \sum_{j: j \neq i} \frac{1}{\left|\lambda_{j}-\lambda_{i}\right|^{2}} .
$$

This quantity plays an important role in Ref. 33, where it was observed that it captures quantitatively the derivatives of $\lambda_{i}(A)$. Here, we write it in terms of the Green function and prove it is stable under DBM (2.15). As a result, based on the idea of Green function comparison, it can be used to derive the weak level repulsion estimate Theorem 4.1, once we know such an estimate for larger times, see Theorem 4.4.

Since $Q_{i}(A)$ is not well-defined on the space of real symmetric matrices (it will blow up when $\lambda_{i}(A)$ is not a single eigenvalue), we have to compose it with a cutoff function $\chi_{M}$, where $M:=N^{2 \tau}$ and the (small) constant $\tau>0$ will be chosen later. We choose the cutoff function $\chi_{M}(x)$ which satisfies the following two properties: (1) $\chi_{M}$ is smooth, and the first three derivatives are bounded by some constant $C$, i.e., $\left|\chi_{M}^{\prime}(x)\right|,\left|\chi_{M}^{\prime \prime}(x)\right|,\left|\chi_{M}^{\prime \prime \prime}(x)\right| \leq C$. (2) On the interval $[0, M],\left|\chi_{M}(x)-x\right| \leq 1$, and for $x \geq M, \chi_{M}(x)=M$.

If $\lambda_{i}$ is a single eigenvalue of $A$, then in a neighborhood of $A, \chi_{M}\left(Q_{i}(A)\right)$ is smooth; if $\lambda_{i}$ is not a single eigenvalue of $A$, then in a neighborhood of $A, \chi_{M}\left(Q_{i}(A)\right)$ is constant, which is also smooth. Therefore, $\chi_{M}\left(Q_{i}(A)\right)$ is a well defined smooth function on the space of real symmetric matrices.

Our proof of Theorem 4.1 consists of three steps:

1. We use the level repulsion estimate for $H_{t}$ to conclude the estimate $\mathbb{E}\left[\chi_{M}\left(Q_{i}\left(H_{t}\right)\right)\right] \leq C N^{3 \tau / 2}$ for $t=N^{-1+\epsilon}$.

2. We compare $\mathbb{E}\left[\chi_{M}\left(Q_{i}\left(H_{t}\right)\right)\right]$ for $t=N^{-1+\epsilon}$ and $\mathbb{E}\left[\chi_{M}\left(Q_{i}\left(H_{0}\right)\right)\right]$ using the continuity Lemma 4.3. Since $\epsilon>0$ can be chosen arbitrarily small, we will obtain

$$
\left|\mathbb{E}\left[\chi_{M}\left(Q_{i}\left(H_{t}\right)\right)\right]-\mathbb{E}\left[\chi_{M}\left(Q_{i}\left(H_{0}\right)\right)\right]\right| \leq C N^{\tau} .
$$

3. Theorem 4.1 then immediately follows from $\mathbb{E}\left[\chi_{M}\left(Q_{i}\left(H_{0}\right)\right)\right] \leq C N^{3 \tau / 2}$ and the Markov inequality.

In the remainder of this section, we denote the eigenvalues of $H_{t}$ by $\lambda_{1}(t) \leq \lambda_{2}(t) \leq \cdots \leq \lambda_{N}(t)$, and so, the eigenvalues of $H$ are $\lambda_{1}(0) \leq \lambda_{2}(0) \leq \cdots \leq \lambda_{N}(0)$.

The following level repulsion estimate is an immediate consequence of Ref. 26 (Theorem 3.6).

Theorem 4.4. Let $H$ be a stable random matrix as defined in Section II C. Given any (small) number $\omega>0$, and (large) number $D>0$, we have that

$$
\mathbb{P}\left(\left|\lambda_{i}(t)-\lambda_{i+1}(t)\right| \leq \theta N^{-1}\right) \leq N^{\omega} \theta^{2-\omega}+N^{-D}
$$

for any $i \in[[\kappa N,(1-\kappa) N]]$ and $t \geq N^{-1+\epsilon}$.

From this, we derive the following estimate.

Lemma 4.5. Let $H$ be a stable random matrix. Then,

$$
\mathbb{E}\left[\chi_{M}\left(Q_{i}\left(H_{t}\right)\right)\right] \leq C N^{3 \tau / 2}
$$

for $t=N^{-1+\epsilon}$ for any $\epsilon>0$ and all small $\tau>0$.

Proof. By our assumption with probability larger than $1-N^{-D}$, the matrix $H_{t}$ is $\delta$-general in the sense of Definition 2.3. Combining this with Theorem 4.4, we have

$$
\mathbb{P}\left(H_{t} \text { is } \delta \text {-general and }\left|\lambda_{i}(t)-\lambda_{i \pm 1}(t)\right| \geq \theta N^{-1}\right) \geq 1-N^{\omega} \theta^{2-\omega}-N^{-D} .
$$


Define a dyadic decomposition

$$
\begin{aligned}
& U_{0}=\left\{j \neq i:\left|\lambda_{j}(t)-\lambda_{i}(t)\right| \leq N^{-1+\delta}\right\}, \quad U_{\infty}=\left\{j: N^{\delta}<\left|\lambda_{j}(t)-\lambda_{i}(t)\right|\right\}, \\
& U_{n}=\left\{j: 2^{n-1} N^{-1+\delta}<\left|\lambda_{j}(t)-\lambda_{i}(t)\right| \leq 2^{n} N^{-1+\delta}\right\}, \quad 1 \leq n \leq\left\lceil\log _{2} N\right\rceil .
\end{aligned}
$$

For those $H_{t}$ which are $\delta$-general in the sense of Definition 2.3, $\left|U_{n}\right| \leq C 2^{n} N^{\delta}$, for $0 \leq n \leq$ $\left\lceil\log _{2} N\right\rceil$. On the event that $H_{t}$ is $\delta$-general and $\left|\lambda_{i}(t)-\lambda_{i \pm 1}(t)\right| \geq \theta N^{-1}$, we derive the estimate,

$$
Q_{i}\left(H_{t}\right)=\frac{1}{N^{2}} \sum_{n=0}^{\left\lceil\log _{2} N\right\rceil} \sum_{j \in U_{n}} \frac{1}{\left|\lambda_{i}(t)-\lambda_{j}(t)\right|^{2}}+\frac{1}{N^{1+2 \delta}} \leq 3 C N^{\delta} \theta^{-2} .
$$

Therefore, we have

$$
\mathbb{P}\left(\frac{1}{N^{2}} \sum_{j: j \neq i} \frac{1}{\left|\lambda_{i}(t)-\lambda_{j}(t)\right|^{2}} \geq 3 C N^{\delta} \theta^{-2}\right) \leq N^{\omega} \theta^{2-\omega}+N^{-D},
$$

where $\omega>0$ can be any small number. Therefore,

$$
\mathbb{E}\left[\chi_{M}\left(Q_{i}\left(H_{t}\right)\right)\right] \leq 1+3 C N^{\delta} \theta^{-2}+M\left(N^{\omega} \theta^{2-\omega}+N^{-D}\right) .
$$

If we take $\theta=N^{-\tau / 2}$, then we get $\mathbb{E}\left[\chi_{M}\left(Q_{i}\left(H_{t}\right)\right)\right] \leq C N^{3 \tau / 2}$, if we take $\delta<\tau / 2, \omega<\tau / 4$ and $D$ large.

This finishes the proof of the first step. In order to apply Lemma 4.3 for the second step, we need to control the third derivative of $\chi_{M}\left(Q_{i}\left(\theta^{a b} H_{s}\right)\right)$.

Proposition 4.6. Let $A$ be an $N \times N$ deterministic real symmetric matrix. If $A$ is $\delta$-general in the sense of Definition 2.3 and

$$
Q_{i}(A)=\frac{1}{N^{2}} \sum_{j: j \neq i} \frac{1}{\left|\lambda_{i}(A)-\lambda_{j}(A)\right|^{2}} \leq M=N^{2 \tau},
$$

then

for some constant $C$.

$$
\partial_{a b}^{(k)} Q_{i}(A) \leq C N^{(2 k+2) \delta+(k+2) \tau}, \quad k=1,2,3
$$

Proof. We denote $G=(A-z)^{-1}$ the resolvent of $A$ and by $\lambda_{j}$ and $u_{j}$ the eigenvalues and eigenvectors of $A$. Notice that (4.9) implies $\left|\lambda_{i}-\lambda_{i \pm 1}\right| \geq N^{-1-\tau}$. The same dyadic argument leading to (4.8) yields

$$
\sum_{j: j \neq i} \frac{1}{\left|\lambda_{i}-\lambda_{j}\right|}=\sum_{n \geq 0} \sum_{j \in U_{n}} \frac{1}{\left|\lambda_{i}-\lambda_{j}\right|} \leq C N^{1+\tau+\delta} .
$$

Also we have the trivial bound for higher moments

$$
\sum_{j: j \neq i} \frac{1}{\left|\lambda_{i}-\lambda_{j}\right|^{k}} \leq\left(\sum_{j: j \neq i} \frac{1}{\left|\lambda_{i}-\lambda_{j}\right|^{2}}\right)^{k / 2} \leq N^{k(1+\tau)}, \quad k \geq 2 .
$$

We denote by $V$ the matrix whose matrix elements are zero everywhere except at the $(a, b)$ and $(b, a)$ positions, where it equals one. From formula (4.3),

$$
\partial_{a b}^{(k)} Q_{i}(A)=\frac{1}{N^{2}} \operatorname{Tr} \partial_{a b}^{(k)} \oint \frac{G}{\lambda_{i}-z} d z \oint \frac{G}{\lambda_{i}-w} d w .
$$

Notice that $\partial_{a b}^{(k)} G=(-1)^{k} k !(G V)^{k} G$. By the Leibniz rule, (4.13) can be written as a sum of terms in the following form:

$$
\frac{1}{N^{2}} \operatorname{Tr} \oint(G V)^{k_{1}} G \partial_{a b}^{\left(k_{2}\right)} \frac{1}{\lambda_{i}-z} d z \oint(G V)^{k_{3}} G \partial_{a b}^{\left(k_{4}\right)} \frac{1}{\lambda_{i}-w} d w,
$$

where $k_{1}+k_{2}+k_{3}+k_{4}=k$. We will only prove (4.10) for $k=3$; that is, we will prove 


$$
\left|\partial_{a b}^{(3)} Q_{i}(A)\right| \leq C N^{5 \tau+8 \delta}
$$

The computations for $k=1,2$ are much easier. To evaluate (4.14), we need to compute the first three derivatives of $\lambda_{i}(A)$ with respect to the $(a, b)$ th entry of $A$. We use the following formula to compute the derivatives of $\lambda_{i}$ :

$$
\lambda_{i}=-\frac{1}{2 \pi i} \operatorname{Tr} \oint \frac{z}{A-z} d z
$$

where the contour encloses only $\lambda_{i}$. The $k$ th derivative with respect to $(a, b)$ th entry is

$$
\partial_{a b}^{(k)} \lambda_{i}=-\frac{1}{2 \pi i} \operatorname{Tr} \oint z \partial_{a b}^{(k)} G d z=\frac{(-1)^{k+1} k !}{2 \pi i} \operatorname{Tr} \oint z(G V)^{k} G d z .
$$

For $k=1,2,3$, respectively, we have

$$
\begin{aligned}
\partial_{a b} \lambda_{i} & =\frac{1}{2 \pi i} \sum_{j=1}^{N} \oint \frac{z u_{j}^{*} V u_{j}}{\left(\lambda_{j}-z\right)^{2}} d z=u_{i}^{*} V u_{i}, \\
\partial_{a b}^{(2)} \lambda_{i} & =\frac{-2}{2 \pi i} \sum_{j_{1}, j_{2}} \oint \frac{z\left(u_{j_{1}} V u_{j_{2}}^{*}\right)^{2}}{\left(\lambda_{j_{1}}-z\right)^{2}\left(\lambda_{j_{2}}-z\right)}=2 \sum_{j: j \neq i} \frac{\left(u_{i}^{*} V u_{j}\right)^{2}}{\lambda_{i}-\lambda_{j}}, \\
\partial_{a b}^{(3)} \lambda_{i} & =\frac{6}{2 \pi i} \sum_{j_{1}, j_{2}, j_{3}} \oint \frac{z\left(u_{j_{1}} V u_{j_{2}}^{*}\right)\left(u_{j_{2}} V u_{j_{3}}^{*}\right)\left(u_{j_{3}} V u_{j_{1}}^{*}\right)}{\left(\lambda_{j_{1}}-z\right)^{2}\left(\lambda_{j_{2}}-z\right)\left(\lambda_{j_{3}}-z\right)} \\
& =6 \sum_{\substack{j_{1}, j_{2}: \\
j_{1}, j_{2} \neq i}} \frac{\left(u_{i}^{*} V u_{j_{1}}\right)\left(u_{j_{1}}^{*} V u_{j_{2}}\right)\left(u_{j_{2}}^{*} V u_{i}\right)}{\left(\lambda_{i}-\lambda_{j_{1}}\right)\left(\lambda_{i}-\lambda_{j_{2}}\right)}-6\left(u_{i}^{*} V u_{i}\right) \sum_{j: j \neq i} \frac{\left(u_{i}^{*} V u_{j}\right)\left(u_{j}^{*} V u_{i}\right)}{\left(\lambda_{i}-\lambda_{j}\right)^{2}} .
\end{aligned}
$$

Some straightforward but tedious integration similar to (4.16)-(4.18) reveals that $\partial_{a b}^{(3)} Q_{i}(A)$ is a sum of the following terms (for simplicity of notation, we write $V_{j k}:=u_{j}^{*} V u_{k}$ ):

$$
\begin{aligned}
& \sum_{j: j \neq i} \frac{\Omega}{\left(\lambda_{i}-\lambda_{j}\right)^{5}}, \quad \Omega=V_{i i}^{3}, V_{i i}^{2} V_{j j}, V_{i i} V_{j j}^{2}, V_{j j}^{3}, \\
& \sum_{\substack{j_{1}, j_{2}: \\
j_{1}, j_{2} \neq i}} \frac{\Omega}{\left(\lambda_{i}-\lambda_{j_{1}}\right)^{4}\left(\lambda_{i}-\lambda_{j_{2}}\right)}, \quad \Omega=V_{i i} V_{j_{1} j_{2}}^{2}, V_{j_{1} j_{1}} V_{i j_{2}}^{2}, V_{j_{1} j_{1}} V_{j_{1} j_{2}}^{2}, V_{i i} V_{i j_{2}}^{2}, \\
& \sum_{\substack{j_{1}, j_{2}: \\
j_{1}, j_{2} \neq i}} \frac{\Omega}{\left(\lambda_{i}-\lambda_{j_{1}}\right)^{3}\left(\lambda_{i}-\lambda_{j_{2}}\right)^{2}}, \quad \Omega=V_{j_{2} j_{2}} V_{j_{1} j_{2}}^{2}, V_{i i} V_{i j_{1}}^{2}, V_{i i} V_{j_{1} j_{2}}^{2}, V_{j_{1} j_{1}} V_{j_{1} j_{2}}^{2}, \\
& \sum_{\substack{j_{1}, j_{2}, j_{3}: \\
j_{1}, j_{2}, j_{3} \neq i}} \frac{V_{j_{1} j_{2}} V_{j_{2} j_{3}} V_{j_{3} j_{1}}}{\left(\lambda_{i}-\lambda_{j_{1}}\right)^{3}\left(\lambda_{i}-\lambda_{j_{2}}\right)\left(\lambda_{i}-\lambda_{j_{3}}\right)}, \sum_{\substack{j_{1}, j_{2}, j_{3}: \\
j_{1}, j_{2}, j_{3} \neq i}} \frac{V_{j_{1} j_{2}} V_{j_{2} j_{3}} V_{j_{3} j_{1}}}{\left(\lambda_{i}-\lambda_{j_{1}}\right)^{2}\left(\lambda_{i}-\lambda_{j_{2}}\right)^{2}\left(\lambda_{i}-\lambda_{j_{3}}\right)} .
\end{aligned}
$$

Indeed, if one takes a close look at expression (4.14), the only singularity enclosed by our contour is $\lambda_{i}$. Therefore, by Cauchy's formula, the integral is sum of terms with denominators: $\prod_{j}\left(\lambda_{i}-\lambda_{j}\right)$, as appearing in the above expressions.

Since the eigenvectors of $A$ are completely delocalized, we see that

$$
\left|V_{j k}\right| \leq C N^{-1+\delta} \text {. }
$$

This together with bounds (4.11) and (4.12) yields (4.15)

\section{A. Proof of Theorem 4.1}

By explicit computation, we have

$$
\partial_{a b}^{(3)}\left(\chi_{M}\left(Q_{i}\left(\theta^{a b} H_{s}\right)\right)\right)=\chi_{M}^{\prime \prime \prime}\left(\partial_{a b} Q_{i}\right)^{3}+3 \chi_{M}^{\prime \prime} \partial_{a b}^{(2)} Q_{i} \partial_{a b} Q_{i}+\chi_{M}^{\prime} \partial_{a b}^{(3)} Q_{i}
$$

Notice that the right hand side of (4.20) vanishes unless $Q_{i}\left(\theta^{a b} H_{s}\right) \leq M$. Since $H$ is stable, for any choice of $\theta_{a b}^{a b} \in[0,1], \theta^{a b} H_{s}$ satisfies all the assumptions in Lemma 4.10, with probability larger 
than $1-N^{-D}$ for any large number $D$. Therefore,

$$
\mathbb{P}\left(\sup _{\theta^{a b}}\left|\partial_{a b}^{(3)}\left(\chi_{M}\left(Q_{i}\left(\theta^{a b} H_{s}\right)\right)\right)\right| \leq C N^{12 \delta+9 \tau}\right) \geq 1-N^{-D} .
$$

On the complement of the above event, we will use the following deterministic bound:

$$
\left|\partial_{a b}^{(3)}\left(\chi_{M}\left(Q_{i}\left(\theta^{a b} H_{s}\right)\right)\right)\right| \leq C N^{3+9 \tau} .
$$

We want to apply Lemma 4.3 with $F=\chi_{M} \circ Q_{i}$. For this choice of $F$, we can take $B$ to satisfy

$$
B \leq \sup _{\substack{0 \leq s \leq t \\ 1 \leq i, j \leq N}} \mathbb{E}\left[\left(N^{2}\left|h_{i j}(s)-f\right|^{3}+N\left|h_{i j}(s)-f\right|\right) C N^{12 \delta+9 \tau}\right]+C N^{3+9 \tau} N^{-D} \leq C N^{1+12 \delta+9 \tau-\alpha},
$$

where the last factor $N^{-\alpha}$ is from the third moment of $h_{i j}(s)$. We conclude that

$$
\left|\mathbb{E}\left[\chi_{M}\left(Q_{i}\left(H_{t}\right)\right)\right]-\mathbb{E}\left[\chi_{M}\left(Q_{i}\left(H_{0}\right)\right)\right]\right| \leq C t N^{1+12 \delta+9 \tau-\alpha}=C N^{\epsilon+12 \delta+9 \tau-\alpha} .
$$

Since the two numbers $\epsilon, \delta>0$ can be arbitrarily small, therefore, we can choose $\tau=(\alpha-\epsilon-$ $12 \delta) / 8$ and (4.21) simplifies to

$$
\left|\mathbb{E}\left[\chi_{M}\left(Q_{i}\left(H_{t}\right)\right)\right]-\mathbb{E}\left[\chi_{M}\left(Q_{i}\left(H_{0}\right)\right)\right]\right| \leq C N^{\tau} .
$$

Hence,

$$
\mathbb{E}\left[\chi_{M}\left(Q_{i}\left(H_{0}\right)\right)\right] \leq C N^{3 \tau / 2}
$$

and the proof is easily concluded by the Markov inequality.

\section{BULK UNIVERSALITY OF STABLE RANDOM MATRICES}

In this section, we prove bulk universality for stable random matrices $H$, i.e., Theorem 2.8 by comparing the local statistics between $H$ and $H_{t}$. This will yield the theorem as Theorem 3.1 shows that the latter ensemble exhibits bulk universality. In the following of this section, we denote the eigenvalues of $H_{t}$ by $\lambda_{1}(t) \leq \lambda_{2}(t) \leq \cdots \leq \lambda_{N}(t)$. And so the eigenvalues of $H$ are $\lambda_{1}(0) \leq \lambda_{2}(0) \leq \cdots \leq \lambda_{N}(0)$.

We will obtain the gap universality of $H$ from the more general comparison result below.

Lemma 5.1. Let $H$ be a stable random matrix and let $H_{t}$ be defined as in (2.15). Take $t=$ $N^{-1+\epsilon}$. Then for $\epsilon>0$ small enough, we have

$$
\lim _{N \rightarrow \infty} \mathbb{E}\left[O\left(N \lambda_{i_{1}}(0), \ldots, N \lambda_{i_{n}}(0)\right)\right]-\mathbb{E}\left[O\left(N \lambda_{i_{1}}(t), \ldots, N \lambda_{i_{n}}(t)\right)\right]=0,
$$

for any $i_{1}, i_{2}, \ldots, i_{n} \in[[\kappa N,(1-\kappa) N]]$ and bounded test function $O \in C^{\infty}\left(\mathbb{R}^{n}\right)$ whose first three derivatives are bounded.

Proof. For simplicity of notation, we only state the proof for $n=1$ case, i.e., for any $i \in$ $[[\kappa N,(1-\kappa) N]]$,

$$
\lim _{N \rightarrow \infty} \mathbb{E}\left[O\left(N \lambda_{i}(0)\right)\right]-\mathbb{E}\left[O\left(N \lambda_{i}(t)\right)\right]=0 .
$$

Take a cutoff function $\rho_{M}$ such that $\rho_{M}(x)=1$ for $x \leq M$ and $\rho_{M}(x)=0$ for $x \geq 2 M$, where $M=N^{2 \tau}$ and $\tau>0$ is a small constant. By the level repulsion of $H$ and $H_{t}$ from Sec. IV, we know that

$$
\mathbb{P}\left(Q_{i}\left(H_{s}\right) \geq N^{2 \tau}\right) \leq N^{-\tau / 2}, \quad s=0, t .
$$

Since $O \in C^{\infty}(\mathbb{R})$ is bounded, we have that 


$$
\begin{aligned}
& \left|\mathbb{E}\left[O\left(N \lambda_{i}(0)\right)\right]-\mathbb{E}\left[O\left(N \lambda_{i}(t)\right)\right]\right| \\
\leq & \left|\mathbb{E}\left[O\left(N \lambda_{i}(0)\right) \rho_{M}\left(Q_{i}\left(H_{0}\right)\right)\right]-\mathbb{E}\left[O\left(N \lambda_{i}(t)\right) \rho_{M}\left(Q_{i}\left(H_{t}\right)\right)\right]\right| \\
+ & \|O\|_{\infty}\left(\mathbb{P}\left(Q_{i}\left(H_{0}\right) \geq N^{2 \tau}\right)+\mathbb{P}\left(Q_{i}\left(H_{t}\right) \geq N^{2 \tau}\right)\right) \\
\leq & \left|\mathbb{E}\left[O\left(N \lambda_{i}(0)\right) \rho_{M}\left(Q_{i}\left(H_{0}\right)\right)\right]-\mathbb{E}\left[O\left(N \lambda_{i}(t)\right) \rho_{M}\left(Q_{i}\left(H_{t}\right)\right)\right]\right|+\frac{2\|O\|_{\infty}}{N^{\tau / 2}} .
\end{aligned}
$$

Notice that $O\left(N \lambda_{i}(A)\right) \rho_{M}\left(Q_{i}(A)\right)$ is a well defined smooth function on the space of symmetric functions. Moreover, if the matrix $A$ is $\delta$-general in the sense of Definition 2.3 the same argument as in Proposition 4.6 implies

$$
\left|\partial_{a b}^{(3)} O\left(N \lambda_{i}(A)\right) \rho_{M}\left(Q_{i}(A)\right)\right| \leq C N^{c(\delta+\tau)},
$$

where $c$ and $C$ are constants. Therefore, by Lemma 4.3, we have

$$
\left|\mathbb{E}\left[O\left(N \lambda_{i}(0)\right) \rho_{M}\left(Q_{i}\left(H_{0}\right)\right)\right]-\mathbb{E}\left[O\left(N \lambda_{i}(t)\right) \rho_{M}\left(Q_{i}\left(H_{t}\right)\right)\right]\right| \leq C N^{c(\delta+\tau)+\epsilon-\alpha} \rightarrow 0,
$$

if we take $c(\delta+\tau)+\epsilon<\alpha / 2$.

For the universality of the correlation functions, due to the lack of optimal rigidity, one cannot directly deduce the universality of the correlation functions from (5.1). We need the following Green function comparison lemma, which is similar to previous results in, e.g., Ref. 19 (Theorem 2.3). Previous results are based on moment matching, the proof given here is based on the continuity of DBM (Ref. 6, Lemma A.2).

Lemma 5.2. Let $H$ be a stable random matrix as defined in Section II C, and let $H_{t}$ be defined as in (2.15). Let $\delta>0$ be arbitrary and choose an $\eta$ with $N^{-1-\delta} \leq \eta \leq N^{-1}$. For any sequence of positive integers $k_{1}, k_{2}, \ldots, k_{n}$, any set of complex parameters $z_{j}^{m}=E_{j}^{m} \pm \eta$, where $1 \leq j \leq k_{m}, 1 \leq m \leq n,\left|E_{j}^{m}\right| \leq 2-\kappa$, and the \pm signs are arbitrary, we have the following. Let $G_{t}(z)=\left(H_{t}-z\right)^{-1}$ be the resolvent and let $F\left(x_{1}, x_{2}, \ldots, x_{n}\right)$ be a test function such that for any multi-index $\alpha=\left(\alpha_{1}, \ldots, \alpha_{n}\right)$ with $1 \leq|\alpha| \leq 4$ and for any $\omega>0$ sufficiently small, we have

$$
\max \left\{\left|\partial^{\alpha} F\left(x_{1}, x_{2}, \ldots, x_{n}\right)\right|: \max _{j}\left|x_{j}\right| \leq N^{\omega}\right\} \leq N^{C_{0} \omega}
$$

and

$$
\max \left\{\left|\partial^{\alpha} F\left(x_{1}, x_{2}, \ldots, x_{n}\right)\right|: \max _{j}\left|x_{j}\right| \leq N^{2}\right\} \leq N^{C_{0}}
$$

for some constant $C_{0}$. Then the following holds:

$$
\left|\mathbb{E}\left[F\left(N^{-k_{1}} \operatorname{Tr} \prod_{j=1}^{k_{1}} G_{t}\left(z_{j}^{1}\right), \ldots, N^{-k_{n}} \operatorname{Tr} \prod_{j=1}^{k_{n}} G_{t}\left(z_{j}^{n}\right)\right)-\mathbb{E}\left[F\left(G_{t} \rightarrow G_{0}\right)\right]\right]\right| \leq C t N^{1+c \delta-\alpha},
$$

where $c$ and $C$ are constants depending on $\kappa, n, k_{1}, k_{2}, \ldots, k_{n}$ and $C_{0}$.

Proof. For simplicity of notation, we state the proof only for $n=1$ and $k_{1}=1$ cases, i.e.,

$$
\left|\mathbb{E}\left[F\left(N^{-1} \operatorname{Tr} G_{t}(z)\right)\right]-\mathbb{E}\left[F\left(N^{-1} \operatorname{Tr} G_{0}(z)\right)\right]\right| \leq C t N^{1+c \delta-\alpha} .
$$

We will prove this lemma using Lemma 4.3. We must compute derivatives of the trace of the Green's function of the deformed matrix $\theta^{a b} H_{s}$. We denote the resolvent of $\theta^{a b} H_{s}$ by $G(z)=\left(\theta^{a b} H_{s}-z\right)^{-1}$. For the derivatives of $G(z)$, we have $\left|\operatorname{Tr} \partial_{a b}^{(k)} G\right|=\left|(-1)^{k} k ! \operatorname{Tr}(G V)^{k} G\right|$, where $V$ is the matrix whose matrix elements are zero everywhere except at the $(a, b)$ and $(b, a)$ positions, where it equals one. Since $V$ has at most two nonzero elements, the trace $(G V)^{k} G$ contains at most $2^{k} N$ terms. Furthermore, each term is a product of $k+1$ entries of $G$, e.g., $\operatorname{Tr} G V G=$ $\sum_{k}\left(G_{k b} G_{a b}+G_{k a} G_{b k}\right)$. We first derive a bound on the resolvent entries $G_{j k}(E+i \eta)$ down to 
$\eta \geq N^{-1-\delta}$ when $\theta^{a b} H_{s}$ is $\delta$-general. Using the delocalization of the eigenvectors, we have

$$
\left|G_{j k}(E+i \eta)\right| \leq \sum_{i=1}^{N} \frac{\left|u_{i}(j) u_{i}(k)\right|}{\left|\lambda_{i}-z\right|} \leq C N^{-1+\delta} \sum_{i=1}^{N} \frac{1}{\left|\lambda_{i}-z\right|} .
$$

Define a dyadic decomposition

$$
\begin{aligned}
& U_{0}=\left\{j:\left|\lambda_{j}-E\right| \leq N^{-1+\delta}\right\}, \quad U_{\infty}=\left\{j: N^{\delta}<\left|\lambda_{j}-E\right|\right\}, \\
& U_{n}=\left\{j: 2^{n-1} N^{-1+\delta}<\left|\lambda_{j}-E\right| \leq 2^{n} N^{-1+\delta}\right\}, \quad 1 \leq n \leq\left\lceil\log _{2} N\right\rceil .
\end{aligned}
$$

For $\delta$-general $\theta^{a b} H_{s}$, we have $\left|U_{n}\right| \leq C 2^{n} N^{\delta}$, for $0 \leq n \leq\left\lceil\log _{2} N\right\rceil$. We can divide the summation over $i$ into $\cup_{n} U_{n}$,

$$
\left|G_{j k}(E+i \eta)\right| \leq C N^{-1+\delta} \sum_{n \geq 0} \sum_{i \in U_{n}} \frac{1}{\left|\lambda_{i}-E-i \eta\right|} \leq C N^{3 \delta}, \quad N^{-1-\delta} \leq \eta .
$$

Therefore, we have

$$
\mathbb{P}\left(N^{-1} \sup _{\theta^{a b}}\left|\operatorname{Tr} \partial_{a b}^{(k)} G\right| \leq C N^{3(k+1) \delta}\right) \geq 1-N^{-D}, \quad N^{-1-\delta} \leq \eta .
$$

When $\theta^{a b} H_{s}$ is not $\delta$-general, we still have the deterministic upper bound

$$
N^{-1}\left|\operatorname{Tr} \partial_{a b}^{(k)} G\right| \leq C N^{3(1+\delta)}
$$

Therefore, we can take $F(A)=N^{-1} \operatorname{Tr}(A-E-i \eta)^{-1}$ for $N^{-1-\delta} \leq \eta$ in Lemma 4.3. For $B$ we have the upper bound

$$
\begin{aligned}
B & \leq \sup _{0 \leq s \leq t, 1 \leq i, j \leq N} \mathbb{E}\left[\left(N^{2}\left|h_{i j}(s)-f\right|^{3}+N\left|h_{i j}(s)-f\right|\right) C N^{\left(3 C_{0}+18\right) \delta}\right]+C N^{3(1+\delta)} N^{-D} \\
& \leq C N^{1+\left(3 C_{0^{+}}+18\right) \delta-\alpha},
\end{aligned}
$$

which yields (5.5).

Once we have the above lemma, the following theorem from Ref. 14 (Theorem 2.1) transforms the information of the Green function to the correlation functions of $H$ and will complete the proof of Theorem 2.8.

Theorem 5.3. Let $G(z)$ and $G_{t}(z)$ denote the Green function of the two matrices $H$ and $H_{t}$, respectively. Suppose that (5.5) holds for the two random matrices $H$ and $H_{t}$ for any $t=N^{-1+\epsilon}$, where $\epsilon>0$ can be arbitrarily small. Let $\rho_{H}^{(n)}$ and $\rho_{H_{t}}^{(n)}$ be the n-point correlation functions of the eigenvalues with respect to the probability laws of the matrices $H$ and $H_{t}$. Then for any $\kappa>0$, $E \in(-2+\kappa, 2-\kappa)$, and test function $O \in C_{c}^{\infty}\left(\mathbb{R}^{n}\right)$, we have

$$
\lim _{N \rightarrow \infty} \int_{\mathbb{R}^{n}} O\left(\alpha_{1}, \ldots, \alpha_{n}\right)\left\{\rho_{H}^{(n)}\left(E+\frac{\alpha_{1}}{N}, \ldots, E+\frac{\alpha_{n}}{N}\right)-\rho_{H_{t}}^{(n)}\left(E+\frac{\alpha_{1}}{N}, \ldots, E^{\prime}+\frac{\alpha_{n}}{N}\right)\right\}=0
$$

provided that $t$ is chosen so that $C t N^{1+c \delta-\alpha} \leq N^{-\alpha / 2}$.

\section{A. Proof of Theorem 2.8}

The universality of the gap statistics follows from Theorem 3.1 and Lemma 5.1. The universality of the correlation functions follows from Theorem 3.1, Lemma 5.2, and Theorem 5.3.

\section{UNIVERSALITY OF SPARSE RANDOM MATRICES}

In this section, we prove Theorem 2.2, the bulk universality of sparse matrices, by checking that sparse matrices satisfy the hypotheses of Definition 2.4 of stable random matrices. In the following, we collect some facts about sparse matrices proved in Ref. 9. 
Theorem 6.1. Let $H$ be a sparse Wigner matrix as in Definition 2.1. We denote the eigenvalues of $H$ as $\lambda_{1} \leq \lambda_{2} \leq \cdots \leq \lambda_{N}$, the corresponding eigenvectors $u_{1}, u_{2}, \ldots, u_{N}$, and the resolvent $G(E+i \eta)=(H-E-i \eta)^{-1}$. Then for all (small) $\omega, \delta>0$, (large) $D>0$, and large enough $N \geq N(\omega, \delta, D)$, the following holds with probability larger than $1-N^{-D}$.

(i) All eigenvalues of $H$ are in the interval $[-3,3]$, i.e., $-3 \leq \lambda_{1} \leq \lambda_{2} \cdots \leq \lambda_{N-1} \leq 3$, except for the largest eigenvalue $\lambda_{N}$.

(ii) We have the weak local semi-circle law for individual resolvent entries: there exists a constant $C$ such that $\left|G_{j k}(E+i \eta)\right| \leq C$, for $\eta \geq N^{-1+\delta}$.

(iii) We have the local semi-circle law for the Stieltjes transform of eigenvalues of $H$,

$$
\left|m_{N}(E+i \eta)-m_{s c}(E+i \eta)\right| \leq N^{\omega}\left(\frac{1}{q}+\frac{1}{N \eta}\right), \quad|E| \leq 5, N^{-1+\delta} \leq \eta \leq 10 .
$$

Remark 6.2. In fact Theorem 6.1 is still true for generalized sparse matrices, in which we allow the variance of each entry $\mathbb{E}\left[\left(h_{i j}-f\right)^{2}\right]=s_{i j}$ to be different, given that (1) they are of the same order, i.e., there exists some constants $c_{1}$ and $c_{2}$, such that $c_{1} N^{-1} \leq s_{i j} \leq c_{2} N^{-1}$, (2) $\sum_{j=1}^{N} s_{i j}=1$.

Theorem 6.3. Sparse random matrices in the sense of Definition 2.1 are stable. Therefore, Theorem 2.2 holds.

Proof. Conditions (1) and (3) of Definition 2.4, i.e., the independent entries and moment conditions, follow from the definition of a sparse matrix. The fact that $\theta^{a b} H_{s}$ is $\delta$-general, i.e., condition (4), will follow from the bounds (ii) in Theorem 6.1. If the resolvent elements of $\theta^{a b} H_{s}$ are bounded down to the scale $\eta \geq N^{-1+\delta}$ then by taking $E=\lambda_{i}$ and $\eta=N^{-1+\delta}$ in the following identity:

$$
C \geq \operatorname{Im} G_{j j}(E+i \eta)=\sum_{i=1}^{N} \frac{\eta\left|u_{i}(j)\right|^{2}}{\left(\lambda_{i}-E\right)^{2}+\eta^{2}} \geq\left|u_{i}(j)\right|^{2} \eta^{-1},
$$

we see that $\left|u_{i}(j)\right|^{2} \leq C N^{-1+\delta}$ and so the eigenvectors of $\theta^{a b} H_{s}$ are completely delocalized. For any interval $I=[E-\eta, E+\eta]$, such that $N^{-1+\delta} \leq \eta$, we have

$$
C \geq \operatorname{Im} m(E+i \eta)=\frac{1}{N} \sum_{i=1}^{N} \frac{\eta}{\left(\lambda_{i}-E\right)^{2}+\eta^{2}} \geq \frac{1}{N} \sum_{\lambda_{i} \in I} \frac{\eta}{\left(\lambda_{i}-E\right)^{2}+\eta^{2}} \geq \frac{1}{|I| N} \#\left\{i: \lambda_{i} \in I\right\} .
$$

Therefore, we get that $\#\left\{i: \lambda_{i} \in I\right\} \leq C|I| N$ by rearranging the above expression.

It therefore suffices to prove that the resolvent entries of the deformed matrix $\theta^{a b} H_{s}$ are bounded down to the scale $\eta=N^{-1+\delta}$. For each $s, H_{s}$ is a sparse matrix and so by Theorem 6.1 we know that its resolvent entries are bounded with probability greater than $1-N^{-D}$ for any large $D$.

The deformed matrix $\theta^{a b} H_{s}$ is a rank two perturbation of $H_{s}$, i.e., $\theta^{a b} H_{s}=H_{s}-V$, where $V$ is the matrix whose matrix elements are zero everywhere except at the $(a, b)$ and $(b, a)$ positions, where it equals $\left(1-\theta_{a b}^{a b}\right) h_{a b}(s)$. By our assumption on the moments of sparse Wigner matrix (2.9), we have that

$$
\mathbb{P}\left(\left|\left(1-\theta_{a b}^{a b}\right) h_{a b}(s)\right| \leq N^{-\alpha / 2}\right) \geq 1-N^{-D}
$$

for any large $D$. We denote the resolvent of $H_{s}$ as $G=\left(H_{s}-z\right)^{-1}$. The resolvent elements of $\theta^{a b} H_{s}$ are given by the formula

$$
\begin{aligned}
\left|\left(\theta^{a b} H_{s}-z\right)_{j k}^{-1}\right| & =\left|G_{j k}+\left(G V\left(\theta^{a b} H_{s}-z\right)^{-1}\right)_{j k}\right| \\
& \leq C+2 C N^{-\alpha / 2} \max _{1 \leq n, l \leq N}\left|\left(\theta^{a b} H_{s}-z\right)_{n l}^{-1}\right| \\
& \leq C+\frac{1}{2} \max _{1 \leq n, l \leq N}\left|\left(\theta^{a b} H_{S}-z\right)_{n l}^{-1}\right|
\end{aligned}
$$

with probability greater than $1-N^{-D}$, for $N$ sufficiently large. We get $\max _{j, k}\left|\left(\theta^{a b} H_{s}-z\right)_{j k}^{-1}\right| \leq 2 C$, for any choice of $\theta_{a b}^{a b} \in[0,1]$, by taking maximum over $j$ and $k$ in above estimate, and rearranging 
it. This finishes the proof that with probability larger than $1-N^{-D}$ for any (large) number $D, \theta^{a b} H_{s}$ is general, for any choice of $\theta_{a b}^{a b} \in[0,1]$.

For the local semi-circle law of $H_{t}^{(1)}$, i.e., condition (2), the variance of the diagonal terms and off-diagonal terms of $H_{t}^{(1)}$ is $e^{-t} N^{-1}$ and $\left(1+e^{-t}\right) / 2 N$, respectively. Therefore, after normalizing by $\left(1-\left(1-e^{-t}\right) \frac{N+1}{2 N}\right)^{-1}=1+O(t)$, it is a generalized sparse matrix. By (3.10), the normalization factor gives us an error of order at most $O(\sqrt{t})$ : with probability larger than $1-N^{-D}$,

$$
\left|m_{N}^{(1)}(z)-m_{s c}(z)\right| \leq N^{\omega}\left(\frac{1}{q}+\frac{1}{N \eta}+\sqrt{t}\right), \quad|E| \leq 4, N^{-1+\delta} \leq \eta \leq 10,
$$

where $m_{N}^{(1)}(z)$ is the Stieltjes transform of the empirical eigenvalue distribution of $H_{t}^{(1)}$. Therefore, we can take $c(\delta)=\frac{1}{2} \min \left\{\alpha, \delta, \frac{1-\epsilon}{2}\right\}$ in (2.19).

We have checked that sparse random matrices are stable, and so, Theorem 2.2 now follows from Theorem 2.8 .

\section{ACKNOWLEDGMENTS}

The work of B.L. is partially supported by NSERC. The work of H.-T.Y. is partially supported by NSF Grant No. DMS-1307444 and a Simons Investigators fellowship.

${ }^{1}$ Adlam, B. and Che, Z., "Spectral statistics of sparse random graphs with a general degree distribution," preprint arXiv: 1509.03368 (2015).

${ }^{2}$ Ajanki, O., Erdős, L., and Krüger, T., "Quadratic vector equation in the complex upper half plane,” preprint arXiv:1506. 05095 (2015).

${ }^{3}$ Ajanki, O., Erdős, L., and Krüger, T., “Universality for general Wigner-type matrices,” preprint arXiv:1506.05098 (2015).

${ }^{4}$ Biane, P., "On the free convolution with a semi-circular distribution," Indiana Univ. Math. J. 46(3), 705-718 (1997).

${ }^{5}$ Bourgade, P., Erdős, L., Yau, H.-T., and Yin, J., "Fixed energy universality for generalized Wigner matrices," Commun. Pure Appl. Math. (to appear); preprint arXiv:1407.5606 (2014).

${ }^{6}$ Bourgade, P. and Yau, H.-T., "The eigenvector moment flow and local quantum unique ergodicity," preprint arXiv:1312. 1301 (2013).

${ }^{7}$ Dyson, F. J., “A Brownian-motion model for the eigenvalues of a random matrix,” J. Math. Phys. 3(6), 1191-1198 (1962).

${ }^{8}$ Erdős, L., Knowles, A., Yau, H.-T., and Yin, J., "Spectral statistics of Erdős-Rényi graphs II: Eigenvalue spacing and the extreme eigenvalues," Commun. Math. Phys. 314(3), 587-640 (2012).

${ }^{9}$ Erdős, L., Knowles, A., Yau, H.-T., and Yin, J., "Spectral statistics of Erdős-Rényi graphs I: Local semicircle law," Ann. Probab. 41(3B), 2279-2375 (2013).

${ }^{10}$ Erdős, L., Knowles, A., Yau, H.-T., and Yin, J., “The local semicircle law for a general class of random matrices,” Electron. J. Probab. 18(59), 1-58 (2013).

${ }^{11}$ Erdős, L., Péché, S., Ramirez, J. A., Schlein, B., and Yau, H.-T., "Bulk universality for Wigner matrices," Commun. Pure Appl. Math. 63(7), 895-925 (2010).

${ }^{12}$ Erdős, L., Schlein, B., and Yau, H.-T., "Wegner estimate and level repulsion for Wigner random matrices,” Int. Math. Res. Not. 2010(3), 436-479.

${ }^{13}$ Erdős, L., Schlein, B., and Yau, H.-T., "Universality of random matrices and local relaxation flow," Invent. Math. 185(1), 75-119 (2011).

${ }^{14}$ Erdős, L., Schlein, B., Yau, H.-T., and Yin, J., "The local relaxation flow approach to universality of the local statistics for random matrices," Ann. Inst. Henri Poincaré Probab. Stat. 48(1), 1-46 (2012).

${ }^{15}$ Erdős, L. and Schnelli, K., "Universality for random matrix flows with time-dependent density," preprint arXiv:1504. 00650 (2015)

${ }^{16}$ Erdős, L. and Yau, H.-T., "Universality of local spectral statistics of random matrices,” Bull. Am. Math. Soc. 49(3), 377-414 (2012).

${ }^{17}$ Erdős, L. and Yau, H.-T., “Gap universality of generalized Wigner and $\beta$-ensembles,” J. Eur. Math. Soc. 17(8), 1927-2036 (2015).

${ }^{18}$ Erdős, L., Yau, H.-T., and Yin, J., “Universality for generalized Wigner matrices with Bernoulli distribution,” J. Combinatorics 2, 15-81 (2011).

${ }^{19}$ Erdős, L., Yau, H.-T., and Yin, J., "Bulk universality for generalized Wigner matrices," Probab. Theory Relat. Fields 154(1-2), 341-407 (2012).

${ }^{20}$ Erdős, L., Yau, H.-T., and Yin, J., "Rigidity of eigenvalues of generalized Wigner matrices,” Adv. Math. 229(3), 1435-1515 (2012).

${ }^{21}$ Evangelou, S. N. and Economou, E. N., "Spectral density singularities, level statistics, and localization in a sparse random matrix ensemble," Phys. Rev. Lett. 68(3), 361-364 (1992).

${ }^{22}$ Fyodorov, Y. V. and Mirlin, A. D., "Localization in ensemble of sparse random matrices,” Phys. Rev. Lett. 67(15), 2049-2052 (1991).

${ }^{23}$ Gaudin, M., “Sur la loi limite de l'espacement des valeurs propres d'une matrice aleéatoire,” Nucl. Phys. 25, 447-458 (1961). 
${ }^{24}$ Knowles, A. and Yin, J., "Eigenvector distribution of Wigner matrices," Probab. Theory Relat. Fields 155(3-4), 543-582 (2013).

${ }^{25}$ Kühn, R., “Spectra of sparse random matrices,” J. Phys. A 41(29), 295002 (2008).

${ }^{26}$ Landon, B. and Yau, H.-T., "Convergence of local statistics of Dyson Brownian motion," preprint arXiv:1504. 03605 (2015).

${ }^{27}$ Mehta, M. L., Random Matrices (Academic Press, 2004), Vol. 142.

${ }^{28}$ Mirlin, A. D. and Fyodorov, Y. V., “Universality of level correlation function of sparse random matrices,” J. Phys. A 24(10), 2273-2286 (1991).

${ }^{29}$ Nguyen, H., Tao, T., and Vu, V., "Random matrices: Tail bounds for gaps between eigenvalues," preprint arXiv:1504: 00396 (2015).

${ }^{30}$ Reed, M. and Simon, B., Methods of Modern Mathematical Physics. IV. Analysis of Operators (Academic Press [Harcourt Brace Jovanovich Publishers], New York, London, 1978), p. XV+396, ISBN: 0-12-585004-2.

${ }^{31}$ Shcherbina, M. and Tirozzi, B., "Central limit theorem for fluctuations of linear eigenvalue statistics of large random graphs: Diluted regime," J. Math. Phys. 53(4), 043501 (2012).

32 Tao, T. and Vu, V., "Random matrices: Universality of local eigenvalue statistics up to the edge," Commun. Math. Phys. 298(2), 549-572 (2010).

33 Tao, T. and Vu, V., "Random matrices: Universality of local eigenvalue statistics," Acta Math. 206(1), 127-204 (2011).

${ }^{34}$ Wigner, E. P., "Characteristic vectors of bordered matrices with infinite dimensions," Ann. Math. 62, 548-564 (1955). 